\title{
Post-translational modification of CREB-1 decreases collagen I expression by inhibiting the TGF- $\beta 1$ signaling pathway in rat hepatic stellate cells
}

\author{
XIAOLING DENG ${ }^{1}$, LIANG DENG $^{2}$, PEI WANG ${ }^{1}$, CHUNWEI CHENG $^{1}$ and KESHU XU ${ }^{1}$ \\ ${ }^{1}$ Division of Gastroenterology, Union Hospital, Tongji Medical College, Huazhong University of Science and Technology, \\ Wuhan, Hubei 430022; ${ }^{2}$ Department of Gastroenterology, The First Affiliated Hospital of Chongqing Medical University, \\ Chongqing 630014, P.R. China
}

Received October 15, 2015; Accepted October 7, 2016

DOI: $10.3892 / \mathrm{mmr} .2016 .5926$

\begin{abstract}
Liver fibrosis is a reversible wound-healing response to liver damage. Following liver injury, activated hepatic stellate cells (HSCs) cause excessive deposition of collagen and other components of the extracellular matrix, which may eventually impair liver function. Transforming growth factor- $\beta 1$ (TGF- $\beta 1$ ) is critical for the development of liver fibrosis, and acts by activating HSCs, stimulating matrix deposition and inducing other profibrotic responses. Certain studies have demonstrated that the transcription factor, cyclic adenosine monophosphate (cAMP)-response element binding protein-1 (CREB-1) is important in the inhibition of fibrosis; however, the underlying mechanism remains to be elucidated. The present study hypothesized that CREB-1 inhibits fibrosis via blocking the TGF- $\beta 1$ signaling pathway in HSCs. Treatment with the cAMP-elevating agent, forskolin stimulated CREB-1 phosphorylation and transcription activation in HSCs. In addition, treatment with the histone deacetylase inhibitor, trichostatin A extended CREB-1 phosphorylation. The present study used a pRSV-CREB-1 expression vector to upregulate CREB-1 gene expression. The results indicated that activated CREB-1 reduced TGF- $\beta 1$-stimulated expression of collagen I, activated Smad2/3 and upregulated expression of Smad7. In addition, activated CREB- 1 attenuated the activation of extracellular signal-regulated kinase $1 / 2$ induced by TGF- $\beta 1$, expression of Ras homolog gene family, member A (RhoA) and Rho-associated coiled-coil containing protein kinase 1 . Thus, post-translational modification of CREB-1 inhibited the
\end{abstract}

Correspondence to: Professor Keshu $\mathrm{Xu}$, Division of Gastroenterology, Union Hospital, Tongji Medical College, Huazhong University of Science and Technology, 1277 Jiefang Road, Wuhan, Hubei 430022, P.R. China

E-mail: xuzou2015@126.com

Key words: cyclic adenosine monophosphate-responsive element binding protein-1, hepatic stellate cell, phosphorylation, acetylation, transforming growth factor- $\beta 1$ profibrotic effects of TGF- $\beta 1$ in HSCs via Smad-dependent and -independent signaling pathways.

\section{Introduction}

Liver fibrosis is a common pathological process occurring as a result of a variety of liver diseases and lesions. Transforming growth factor (TGF)- $\beta 1$ is an important promoter of liver fibrosis (1), which activates the differentiation of fibroblasts into myofibroblasts and stimulates the latter to produce a large quantity of matrix proteins (2).

In mammals, the TGF- $\beta$ superfamily is composed of three homologous genes, which may interact with each other: TGF- $\beta 1$, TGF- $\beta 2$ and TGF- $\beta 3$. Our previous study demonstrated that TGF- $\beta 3$ may significantly inhibit the expression of TGF- $\beta 1$ and collagen I, the primary protein of the extracellular matrix (3). A further study demonstrated that the TGF- $\beta 3$ promoter contains the cyclic adenosine monophosphate (cAMP)-responsive element (CRE) site (4). CRE binding protein 1 (CREB-1), phosphorylated (p) on Ser-133, combines with this particular site and activates the TGF- $\beta 3$ promoter, inducing the secretion of TGF- $\beta 3$. Therefore, $p$-CREB- 1 is a critical transcription factor in mediating TGF- $\beta 3$ auto-induction (4).

Previous studies have indicated that p-CREB-1 has an inhibitory effect on myocardial and pulmonary fibroses $(5,6)$. In addition, p-CREB-1 may have an antifibrolytic effect in liver. However, the antifibrolytic effect is counteracted by the inactivation of p-CREB-1 through dephosphorylation (7).

Post-translational modifications, including phosphorylation, are the chemical modifications of newly-synthesized polypeptide chains or proteins, which alter their physical and chemical properties and therefore their biological function. Certain studies have demonstrated that acetylation, another post-translational modification, may alter the conformation of a protein and result in a greater biological effect (8). The present study aimed to identify the underlying mechanisms involved in post-translational modifications of CREB-1 in inhibiting TGF- $\beta 1$-mediated liver fibrosis. The results indicated a theoretical basis for clarifying the underlying CREB-1 antifibrolytic mechanisms, and thereby provide a novel therapeutic target for the treatment of liver fibrosis. 


\section{Materials and methods}

Cell culture and reagents. HSC-T6 rat hepatic stellate cells (HSCs) were provided by the Division of Gastroenterology Laboratory of Union Hospital (Wuhan, China). HSCs were cultured in Dulbecco's modified Eagle's medium (DMEM; Gibco; Thermo Fisher Scientific, Inc., Waltham, MA, USA) supplemented with $10 \%$ fetal bovine serum (FBS; Gibco; Thermo Fisher Scientific, Inc.) at $37^{\circ} \mathrm{C}$ in a humidified atmosphere of $5 \% \mathrm{CO}_{2}$. All experiments were conducted when cells were at an exponential stage of growth. Human recombinant TGF- $\beta 1$ was purchased from PeproTech, Inc. (Rocky Hill, NJ, USA) and used at a concentration of $10 \mathrm{ng} / \mathrm{ml}$. The cAMP-elevating agent, forskolin (FSK) was purchased from Beyotime Institute of Biotechnology (Haimen, China) and the histone deacetylase (HDAC) inhibitor, trichostatin A (TSA) was obtained from Sigma-Aldrich; Merck Millipore (Darmstadt, Germany).

Transfection and treatment. HSCs were seeded into 6-well plates at a concentration of $2 \times 10^{5} /$ well. Transfection with pRSV-CREB-1 expression vector or an empty vector (Invitrogen; Thermo Fisher Scientific, Inc.) was conducted when cells reached 70-90\% confluence. Lipofectamine ${ }^{\mathrm{TM}} 2000$ (10 $\mu \mathrm{l}$; Invitrogen; Thermo Fisher Scientific, Inc.) in $250 \mu \mathrm{l}$ Opti-minimal essential medium (Opti-MEM; Gibco; Thermo Fisher Scientific, Inc.) was mixed with $4 \mu \mathrm{g}$ vector in $250 \mu \mathrm{l}$ Opti-MEM and incubated for $20 \mathrm{~min}$ at room temperature to allow formation of the transfection complex, which was then added to each well (500 $\mu \mathrm{l}$ transfection complex in 1,500 $\mu \mathrm{l}$ DMEM). A total of $6 \mathrm{~h}$ following transfection, culture media were replaced with fresh DMEM, and cells were incubated for an additional $18 \mathrm{~h}$. TGF- $\beta 1$, FSK or TSA were subsequently added into different wells, to a final concentration of $10 \mathrm{ng} / \mathrm{ml}$, $10 \mu \mathrm{M}$ and $100 \mathrm{nM}$ respectively. Treated HSCs were incubated for various times and harvested for subsequent experiments.

Reverse transcription-quantitative polymerase chain reaction $(R T-q P C R)$. Total RNA was extracted using TRIzol ${ }^{\circledR}$ reagent (Ambion; Thermo Fisher Scientific, Inc.). The purity and concentration of the extracted RNA was detected by an ultraviolet spectrophotometer (Eppendorf Biophotometer Plus; Thermo Fisher Scientific, Inc.), and reverse-transcribed to cDNA using PrimeScript ${ }^{\mathrm{TM}}$ RTase kit (Takara Biotechnology Co., Ltd., Dalian, China) according to the manufacturer's protocol. qPCR was performed in a total volume of $10 \mu 1$ with $5 \mu \mathrm{l}$ SYBR Green PCR Master mix (Takara Biotechnology Co., Ltd.), $0.5 \mu \mathrm{l}$ of each primer $(0.2 \mu \mathrm{mol}$ in total), $1 \mu \mathrm{l}$ cDNA (1:10 dilution) and $3 \mu \mathrm{l}$ double-distilled water. Primer sequences are presented in Table I. All cDNA samples were tested in duplicate using a StepOne ${ }^{\mathrm{TM}}$ PCR system (Applied Biosystems; Thermo Fisher Scientific, Inc.), with thermocycling conditions as follows: An initial denaturation step of $50^{\circ} \mathrm{C}$ for $2 \mathrm{~min}$ and $95^{\circ} \mathrm{C}$ for $10 \mathrm{~min}$, followed by 40 cycles of denaturation at $95^{\circ} \mathrm{C}$ for $15 \mathrm{sec}$ and annealing at $60^{\circ} \mathrm{C}$ for $1 \mathrm{~min}$. Fluorescence data from each sample was analyzed using the $2^{-\Delta \Delta C q}$ method (9), with GAPDH gene expression serving as an endogenous control.

Extraction of nuclear protein. Nuclear proteins from HSCs cultured in $100-\mathrm{mm}$ culture dishes were extracted using a
Nuclear-Cytosol Extraction kit (Applygen Technologies, Inc., Beijing, China). Cells were washed with cold PBS three times, lysed in $300 \mu \mathrm{l}$ cytoplasm extraction buffer A for $10 \mathrm{~min}$ and 20-30 $\mu \mathrm{l}$ cytoplasm extraction buffer B for $1 \mathrm{~min}$. Following a 5 min centrifugation at $1,000 \times \mathrm{g}$, the pellet containing nuclei was collected and resuspended in 70-100 $\mu 1$ nuclear extraction buffer for $30 \mathrm{~min}$. The solution was centrifuged at $12,000 \mathrm{x} g$ for $10 \mathrm{~min}$, and the supernatant containing nuclear proteins was collected and stored at $-70^{\circ} \mathrm{C}$ until analysis. All procedures were carried out at $4^{\circ} \mathrm{C}$. Protein concentrations were determined using a Bicinchoninic Acid Protein assay kit (Pierce; Thermo Fisher Scientific, Inc.).

Western blotting. Whole-cell lysates were obtained using radioimmunoprecipitation buffer (Applygen Technologies, Inc.) containing protein phosphatase inhibitor (Applygen Technologies, Inc.), according to the manufacturers' protocol; the nuclear extracts were obtained as described above. Equal quantities of protein $(30-50 \mu \mathrm{g})$ from the lysates were separated by $10 \%$ SDS-PAGE and transferred to polyvinylidene difluoride membranes. Membranes were blocked with 5\% non-fat dry milk in TBS containing $0.1 \%$ Tween-20 (TBST) for $1 \mathrm{~h}$ at room temperature, and incubated individually with the following primary antibodies: Rabbit anti-CREB-1 (1:1,000; catalog no. 9197; Cell Signaling Technology, Inc., Danvers, MA, USA), rabbit anti-mothers against decapentaplegic (Smad) 3 (1:1,500; catalog no. 1735-1; Epitomics, Burlingame, CA, USA), rabbit anti-extracellular signal-regulated kinase 1/2 (ERK1/2; 1:1,000; catalog no. 4695; Cell Signaling Technology, Inc.), rabbit anti-collagen I (1:5,000; catalog no. ab34710; Abcam, Cambridge, UK) and rabbit anti-GAPDH (1:1,000; catalog no. GTX100118; GeneTex, Inc., Irvine, CA, USA), overnight at $4^{\circ} \mathrm{C}$. Separate membranes were blocked with 5\% bovine serum albumin (MP Biomedicals, Santa Ana, CA, USA) in TBST for $1 \mathrm{~h}$ at room temperature, and incubated with rabbit anti-p-CREB (Ser-133; 1:1,000; catalog no. 9198; Cell Signaling Technology, Inc.), goat anti-p-Smad2/3 (Ser-423/425; 1:100; catalog no. sc-11769; Santa Cruz Biotechnology, Inc., Dallas, TX, USA) and rabbit anti-p-ERK1/2 (Thr-202/Tyr-204; 1:1,000; catalog no. 4370S; Cell Signaling Technology, Inc.). Following three washes in TBST, membranes were incubated with anti-rabbit or anti-goat IgG horseradish peroxidase-conjugated secondary antibodies (1:8,000; catalog nos. 111-035-003 and 305-035-003; Jackson ImmunoResearch Laboratories, Inc., West Grove, PA, USA) for $1 \mathrm{~h}$ at room temperature. Target proteins were detected using an Enhanced Chemiluminescence Detection kit (Thermo Fisher Scientific, Inc.). Densitometric analysis was performed using AlphaView Stand Alone software version 3.4.0 (ProteinSimple, San Jose, CA, USA).

Statistical analysis. All experiments were performed three times independently. Data were analyzed using SPSS software version 17.0 (SPSS, Inc., Chicago, IL, USA), using unpaired Student's $t$-tests and one-way analyses of variance followed by Fisher's least significant difference post-hoc test. Data are presented as the mean \pm standard deviation. $\mathrm{P}<0.05$ was considered to indicate a statistically significant difference. Graphs were produced using GraphPad Prism software version 5.0 (GraphPad Software, Inc., La Jolla, CA, USA). 
Table I. Primers used for reverse transcription-quantitative polymerase chain reaction.

\begin{tabular}{lll}
\hline & \multicolumn{2}{c}{ Sequence (5'-3') } \\
\cline { 2 - 3 } Gene & \multicolumn{1}{c}{ Sense } & Antisense \\
\hline CREB-1 & CAGTGCCAACCCCGATTTA & TTGCTCCTCCCTGGGTAATG \\
Collagen I & TTGTGCGATGACGTGATCTGT & TTGGTCGGTGGGTGACTCTG \\
Smad3 & GGGCCTGCTGTCCAATGT & AATGTGCCGCCTTGTAAGCT \\
Smad7 & TGGATGGCGTGTGGGTTTA & TGGCGGACTTGATGAAGATG \\
ERK1/2 & TTTGGTCTGTGGGCTGCAT & TCCTGGGAAGATAGGCCTGTT \\
RhoA & TCGGAATGATGAGCACACAAG & GGTTTACCGGCTCCTGCTT \\
ROCK1 & TTCATTCCTACCCTCTACCACT & GGCTTAAAAACATGCCACAA \\
GAPDH & GTATGACTCTACCCACGGCAAGT & TTCCCGTTGATGACCAGCTT \\
\hline
\end{tabular}

CREB-1, cyclic adenosine monophosphate response element binding protein-1; Smad, mothers against decapentaplegic; ERK1/2, extracellular signal-regulated kinase 1/2; RhoA, Ras homolog gene family member A; ROCK1, Rho-associated coiled-coil containing protein kinase 1.

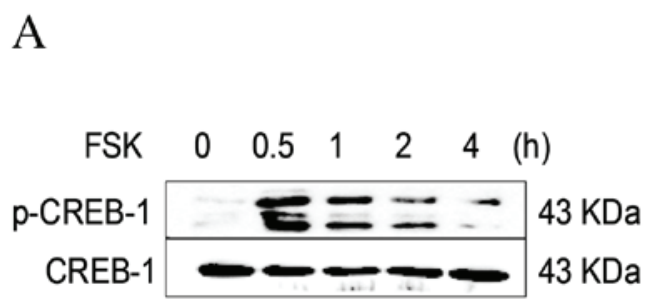

C

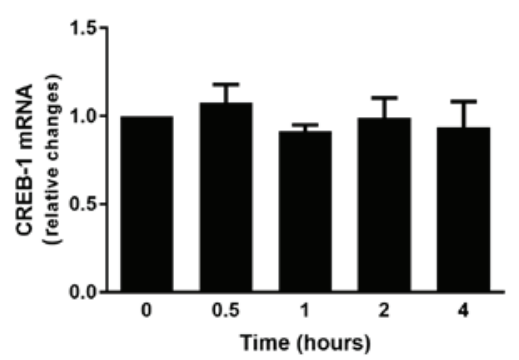

B

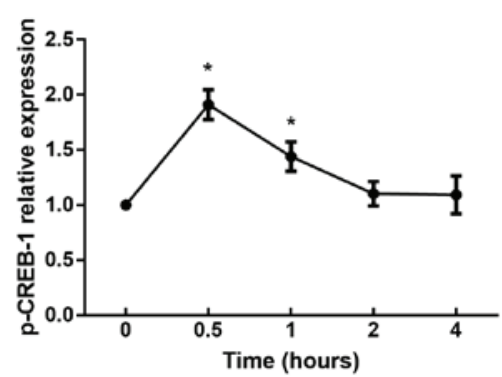

Figure 1. Time-dependent CREB activation by FSK. HSCs were stimulated with $10 \mu \mathrm{M}$ FSK for various times, nuclear proteins were harvested and (A) protein expression of p-CREB-1 and CREB-1 were detected by western blotting. (B) Quantification of western blotting revealed that FSK treatment increased protein expression levels of p-CREB-1 in a time-dependent manner. (C) CREB-1 mRNA was detected by reverse transcription-quantitative polymerase chain reaction and normalized to GAPDH expression. There were no statistically significant differences between the groups at any time points. Data are expressed as the mean \pm standard deviation of at least three independent experiments. * $\mathrm{P}<0.05$ vs. 0 h group. CREB- 1 , cyclic adenosine monophosphate-response element binding protein-1; FSK, forskolin; HSCs, hepatic stellate cells; p, phosphorylated.

\section{Results}

cAMP-elevating agent FSK activates CREB-1. HSCs were stimulated with the cAMP-elevating agent, FSK for various times. The protein expression levels of CREB-1 remained unaltered regardless of the duration of stimulation (Fig. 1A). However, phosphorylation levels of CREB-1 at Ser-133 peaked at $30 \mathrm{~min}$ (1.9-fold greater than control group; $\mathrm{P}=0.011)$, then gradually declined, and maintained a relatively stable level after $1 \mathrm{~h}$ (Fig. 1A and B), demonstrating inconsistencies with a previous study on cardiac fibroblasts (10). In addition, CREB-1 mRNA expression levels following FSK stimulation in HSCs were examined. There were no statistically significant differences between the groups at any time points $(\mathrm{P}=0.863$; Fig. 1C). These results indicate that CREB-1 is activated by FSK in a time-dependent manner.

CREB-1 decreases collagen I expression in HSCs. A pRSV-CREB-1 expression vector and an empty vector were used to transfect HSCs, to investigate whether CREB-1 inhibited collagen I expression. Transfection with pRSV-CREB-1 expression vector increased CREB-1 mRNA expression levels (161.3-fold greater than the empty vector group; $\mathrm{P}<0.001$; Fig. 2A), which was accompanied by a substantial increase in CREB-1 protein expression levels (2.4-fold greater than the empty vector group; $\mathrm{P}=0.003$; Fig. $2 \mathrm{~B}$ and $\mathrm{C}$ ) and a slight 
A

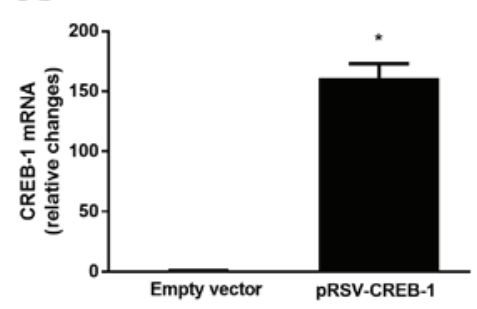

C

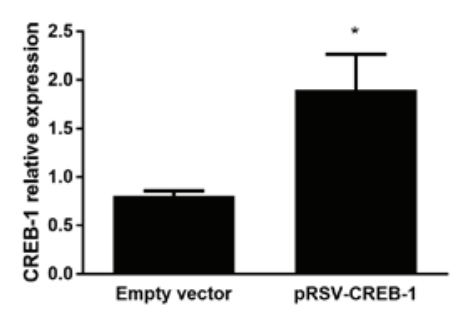

E

a

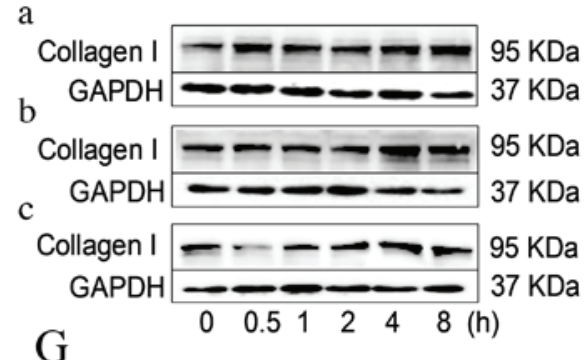

G

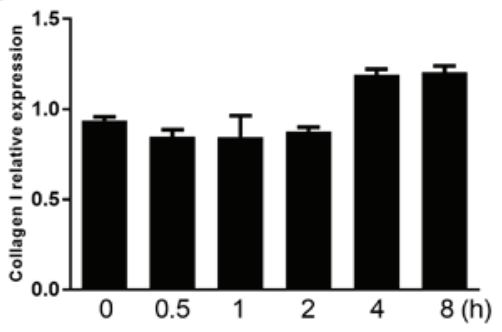

B

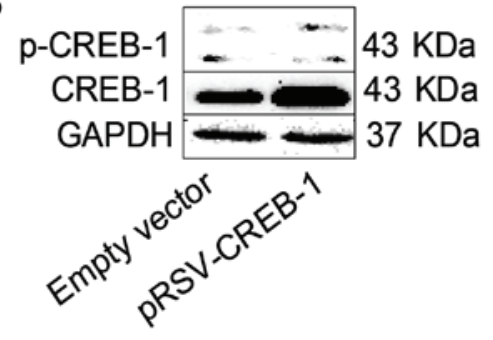

D

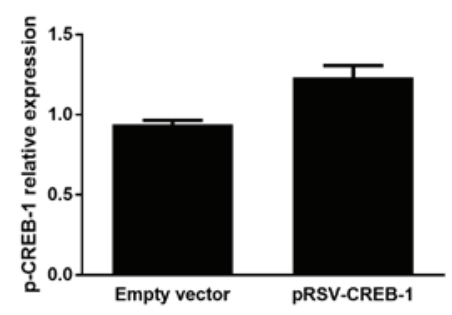

F

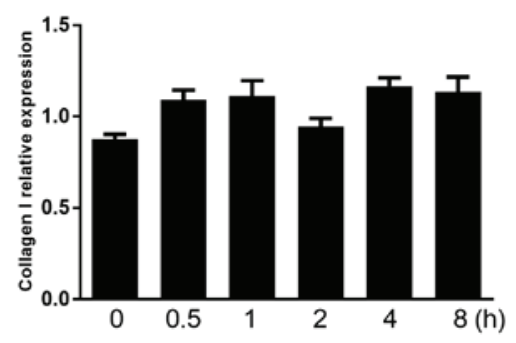

$\mathrm{H}$

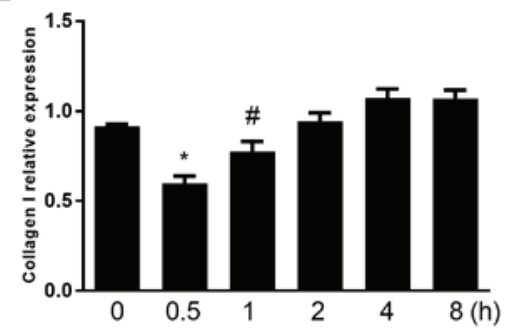

Figure 2. CREB-1 decreases collagen I expression in HSCs. (A-D) HSCs were transfected with pRSV-CREB-1 expression vector or empty vector. A total of $24 \mathrm{~h}$ after transfection (A) CREB-1 mRNA was detected by reverse transcription-quantitative polymerase chain reaction and normalized to GAPDH expression, and (B and C) CREB-1 and (D) p-CREB-1 nuclear protein expression levels were detected by western blotting. Transfection with pRSV-CREB-1 expression vector increased CREB-1 mRNA and protein expression levels, and slightly increased p-CREB-1 protein expression levels, compared with the empty vector control. (E) HSCs were transfected with (a) empty vector, (b) pRSV-CREB-1 expression vector or (c) pRSV-CREB-1 expression vector, followed by treatment with $10 \mu \mathrm{M}$ FSK $24 \mathrm{~h}$ after transfection. Total protein was extracted and collagen I was detected by western blotting. Quantification of (F) empty vector, (G) pRSV-CREB-1 expression vector and (H) pRSV-CREB-1 expression vector followed by FSK treatment. Activated CREB-1 significantly reduced the expression of collagen I with a peak at $0.5 \mathrm{~h}$. The negative effect of CREB-1 on collagen I protein expression levels gradually decreased or was lost over time. Data are presented as the mean \pm standard deviation of at least three independent experiments. (A-D) ${ }^{*} \mathrm{P}<0.01$ vs. empty vector group; (E-H) ${ }^{*} \mathrm{P}<0.05$ and ${ }^{*} \mathrm{P}<0.01$ vs. $0 \mathrm{~h}$ group. CREB-1, cyclic adenosine monophosphate-response element binding protein-1; HSCs, hepatic stellate cells; p, phosphorylated; FSK, forskolin.

increase in p-CREB-1 protein expression ( $\mathrm{P}=0.056$; Fig. 2D). HSCs were treated with FSK 24 h following pRSV-CREB-1 transfection. Activated CREB-1 significantly reduced the expression of collagen I with a peak at $0.5 \mathrm{~h}$ (1.5-fold lower than the empty vector group; $\mathrm{P}=0.003$ ), consistent with the peak of CREB-1 activation. However, the negative effect of CREB-1 on collagen I protein expression levels gradually decreased or was lost over time (Fig. 2E-H).

CREB-1 decreases collagen I expression via inhibiting the Smad-dependent TGF- $\beta 1$ signaling pathway. To elucidate the underlying mechanism involved in CREB-1 inhibition of TGF- $\beta 1$-mediated liver fibrosis, TGF- $\beta 1$ and/or FSK were used to treat transfected cells. As presented in Fig. 3A and B, TGF- $\beta 1$ treatment significantly increased collagen I protein expression levels in the empty vector group $(\mathrm{P}=0.025)$; however, the protein expression levels of collagen I demonstrated only a slight increase under TGF- $\beta 1$ stimulation in the pRSV-CREB-1 expression vector group. In addition, the collagen I expression level was reduced in each pRSV-CREB-1 group compared with the corresponding empty vector group. The proteins of the Smad family were the first identified substrates of type I 
A

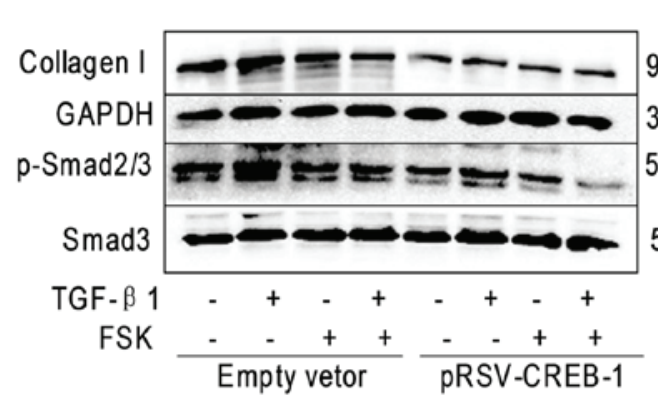

C

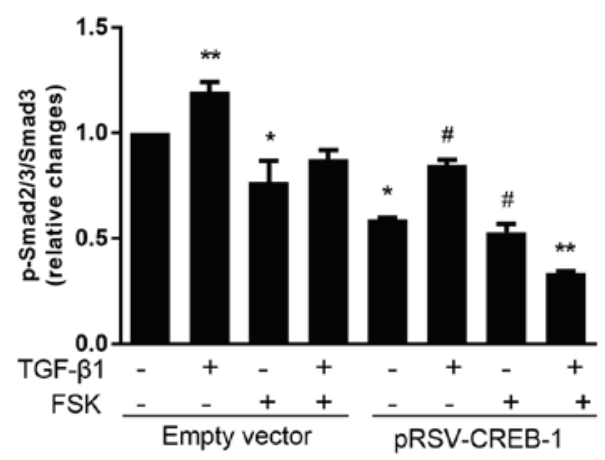

B $95 \mathrm{KDa}$ $37 \mathrm{KDa}$ $55-60 \mathrm{KDa}$ $58 \mathrm{KDa}$

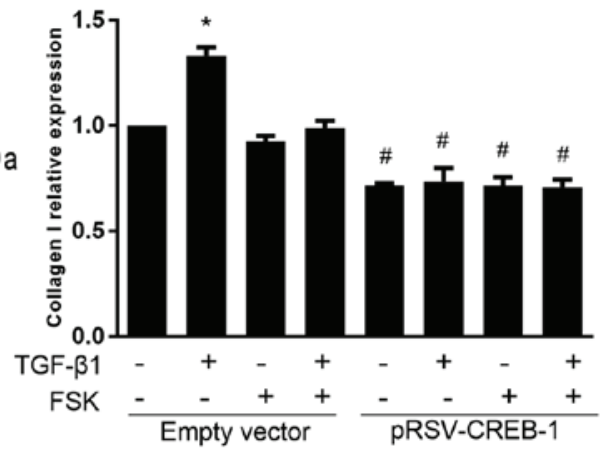

D

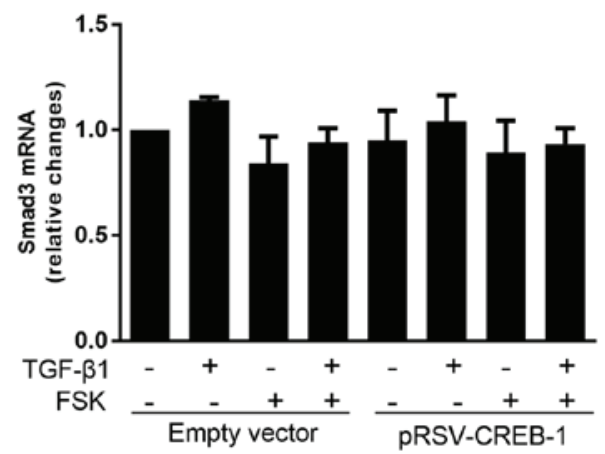

E

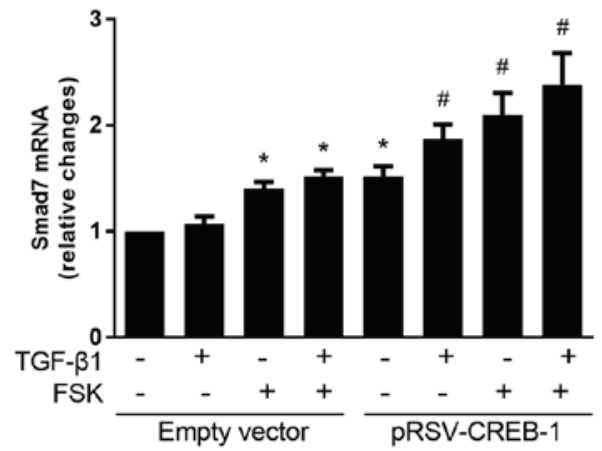

Figure 3. CREB-1 inhibits the TGF- $\beta 1$ signaling pathway via Smad-dependent pathways. Cells were transfected with pRSV-CREB-1 expression vector or empty vector, and $24 \mathrm{~h}$ later were treated with $10 \mu \mathrm{M}$ FSK and/or $10 \mathrm{ng} / \mathrm{ml} \mathrm{TGF}$ - $\beta 1$ for $1 \mathrm{~h}$. (A) Whole-cell lysates were analyzed by western blotting using antibodies against collagen I, p-Smad2/3 (Ser-423/425), Smad3 or GAPDH. (B) TGF- $\beta 1$ significantly increased collagen I protein expression levels in the empty vector group, but not in the pRSV-CREB-1 expression vector group. (C) p-Smad2/3 protein expression levels were consistent with collagen I; however, p-Smad2/3 expression was suppressed in the FSK plus TGF- $\beta 1$-treated group following CREB- 1 overexpression. Total RNA was collected and Smad3 and Smad7 mRNA expression levels were detected by reverse transcription-quantitative polymerase chain reaction. (D) No differences were observed in Smad3 mRNA expression levels between groups. (E) Smad7 mRNA levels were increased following transfection with the pRSV-CREB-1 expression vector, compared with the empty vector control. mRNA results were normalized to GAPDH expression. Data are expressed as the mean \pm standard deviation of at least three independent experiments. ${ }^{*} \mathrm{P}<0.05$ vs. no treatment group; ${ }^{* *} \mathrm{P}<0.05$ vs. all other groups; ${ }^{*} \mathrm{P}<0.05$ vs. corresponding empty vector control group. $\mathrm{CREB}-1$, cyclic adenosine monophosphate-response element binding protein-1; TGF- $\beta 1$, transforming growth factor- $\beta 1$; FSK, forskolin; Smad, mothers against decapentaplegic; p, phosphorylated.

receptor kinases and are key in the TGF- $\beta 1$ signaling pathway. $\mathrm{p}-\mathrm{Smad} 2 / 3$ is considered the final phosphorylation step and the component of the $\mathrm{p}$-Smad complex that translocates to the nucleus to regulate target genes. $\mathrm{p}$-Smad2/3 protein expression levels were consistent with collagen I; however, p-Smad2/3 expression was suppressed significantly in the FSK plus TGF- $\beta 1$-treated group following CREB-1 overexpression $(\mathrm{P}<0.001$; Fig. 3C). No differences were observed in Smad3
mRNA expression levels between groups ( $\mathrm{P}=0.177$; Fig. 3D). The antagonistic Smad7 acts in opposition to p-Smads (11). In the present study, Smad7 mRNA expression levels were greater in the FSK-treated group compared with the TGF- $\beta 1$-treated group (Fig. 3E). In addition, Smad7 mRNA expression levels were greater in each pRSV-CREB-1 group, compared with the respective empty vector control, particularly in the FSK plus TGF- $\beta 1$-treated group $(\mathrm{P}<0.001)$. 

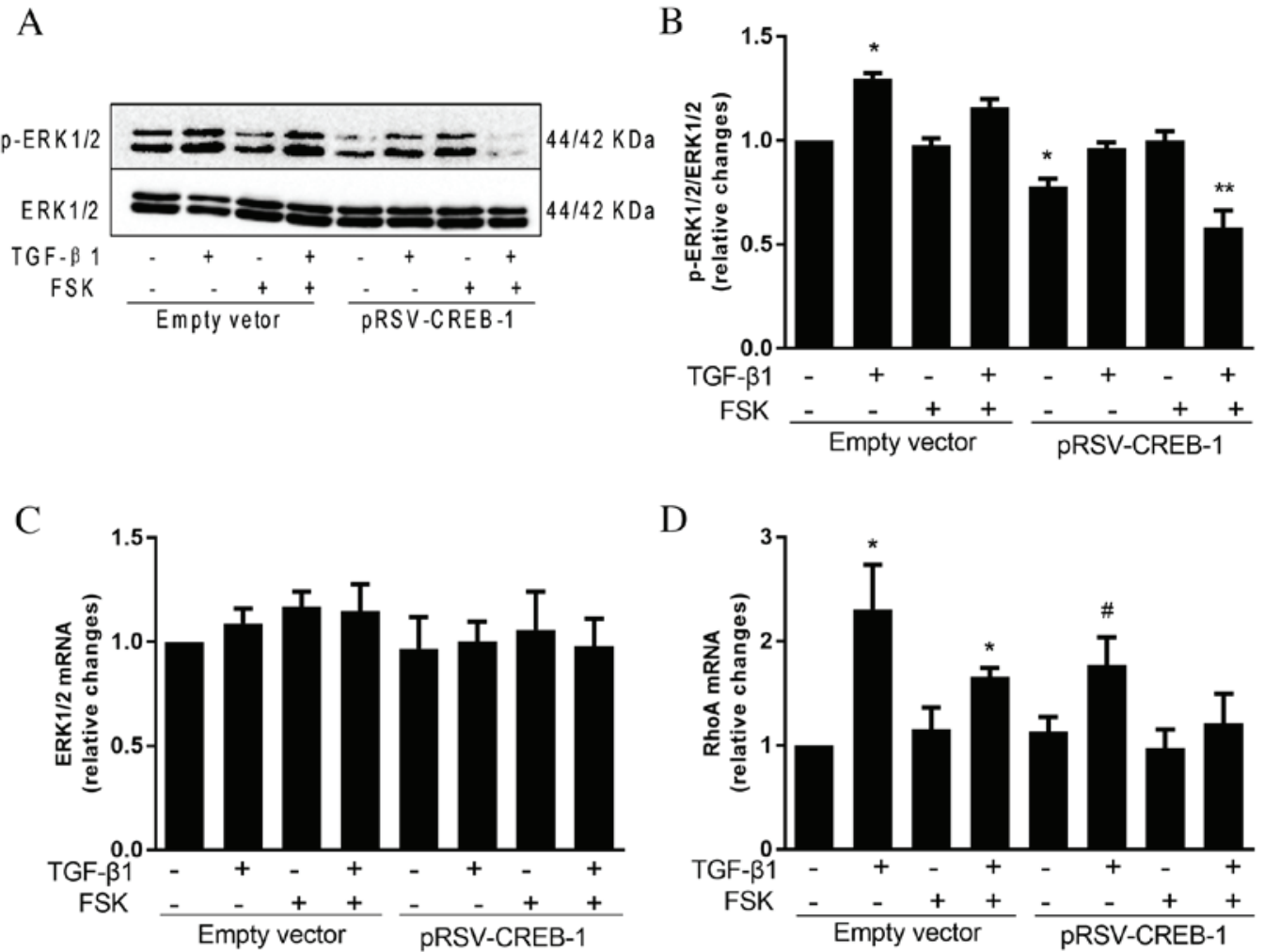

D
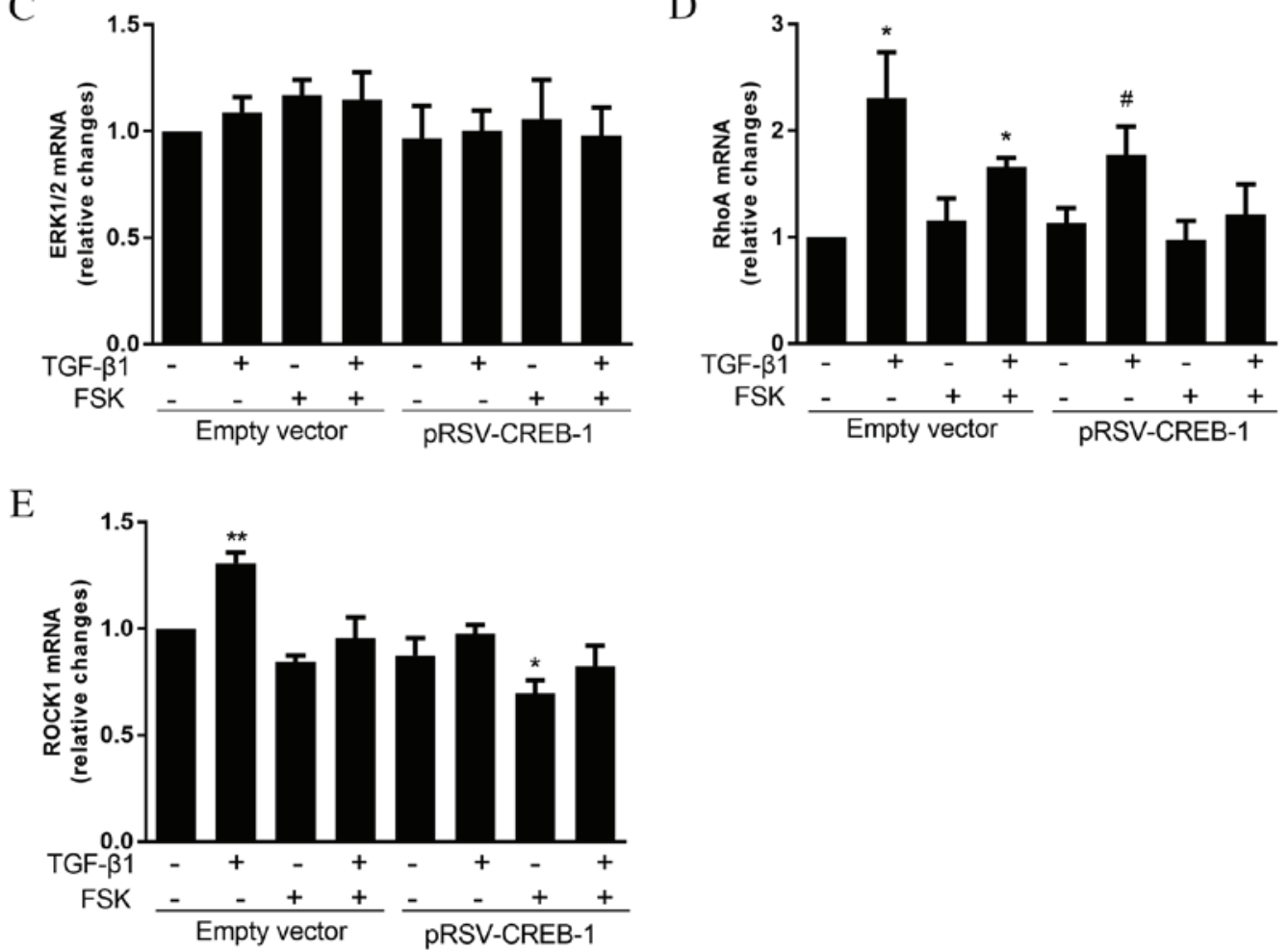

Figure 4. CREB-1 inhibits the TGF- $\beta 1$ signaling pathway via Smad-independent pathways. Cells were transfected with a pRSV-CREB-1 expression vector or an empty vector and $24 \mathrm{~h}$ later treated with $10 \mu \mathrm{M}$ FSK and/or $10 \mathrm{ng} / \mathrm{ml}$ TGF- $\beta 1$ for $1 \mathrm{~h}$. (A) Whole-cell lysates were analyzed by western blotting using antibodies against p-ERK1/2 (Thr-202/Tyr-204) and ERK1/2. (B) Quantification of western blotting revealed that CREB-1 overexpression significantly attenuated the activation of ERK1/2 induced by TGF- $\beta 1$. The total RNA was collected, and (C) ERK1/2, (D) RhoA and (E) ROCK1 mRNA expression levels were detected by reverse transcription-quantitative polymerase chain reaction. TGF- $\beta 1$ stimulated the expression of RhoA and ROCK1; however, CREB-1 overexpression weakened the effect of TGF- $\beta 1$ stimulation. mRNA results were normalized to GAPDH expression. Data are expressed as the mean \pm standard deviation of at least three independent experiments. ${ }^{*} \mathrm{P}<0.05$ vs. no treatment group; ${ }^{* *} \mathrm{P}<0.05$ vs. all other groups; ${ }^{*} \mathrm{P}<0.05$ vs. corresponding empty vector control group. ERK1/2, extracellular signal-regulated kinase 1/2; CREB-1, cyclic adenosine monophosphate response element binding protein-1; FSK, forskolin; TGF- $\beta 1$, transforming growth factor- $\beta 1$; p, phosphorylated; RhoA, Ras homolog gene family member A; ROCK1, Rho-associated coiled-coil containing protein kinase 1.

CREB-1 decreases collagen I expression via inhibition of the Smad-independent TGF- $\beta 1$ signaling pathway. p-ERK1/2 and Ras homolog gene family member A (RhoA)/Rho-associated coiled-coil containing protein kinase 1 (ROCK1) signaling are involved in the TGF- $\beta 1$ signaling pathway in cardiac fibroblasts and corneal myofibroblasts $(5,12)$. These studies indicated that increased cAMP markedly blocks TGF- $\beta 1$-mediated activation of collagen synthesis and $\alpha$-smooth muscle actin expression via inhibition of ERK1/2 phosphorylation and RhoA activation.
The present study hypothesized that activated CREB-1 has a similar role in HSCs. To verify this, p-ERK1/2 and ERK1/2 protein expression levels were analyzed by western blotting, and ERK1/2, RhoA and ROCK1 mRNA expression levels were analyzed via RT-qPCR following CREB-1 overexpression. The results demonstrated inconsistencies with collagen I and p-Smad2/3 data, as p-ERK1/2 levels were slightly, but not significantly, increased by FSK stimulation alone following CREB-1 overexpression $(\mathrm{P}=0.201)$. However, activated 
A

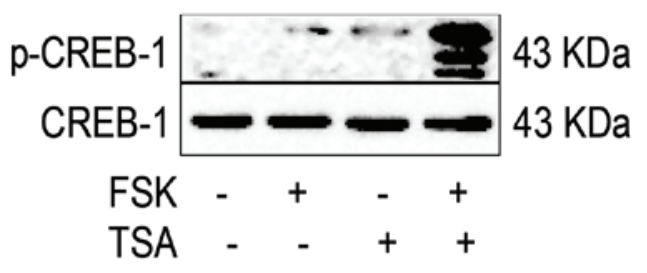

B

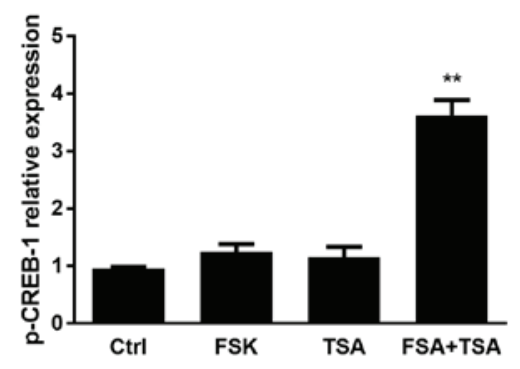

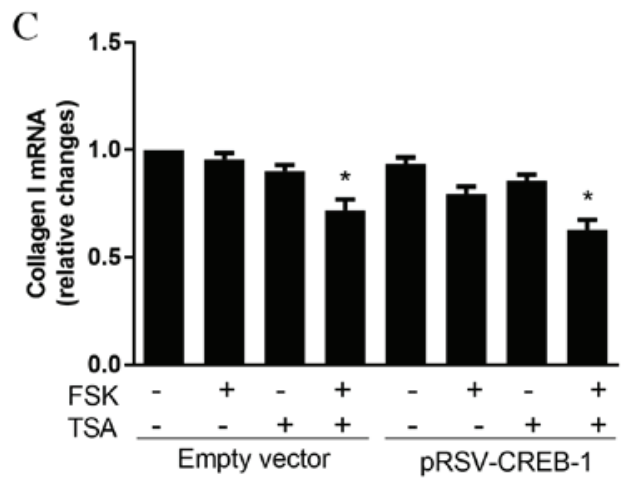

Figure 5. Acetylation extends the function of phosphorylated CREB-1 in decreasing collagen I expression. Cells were stimulated with $10 \mu \mathrm{M}$ FSK and/or $100 \mathrm{nM}$ TSA for $4 \mathrm{~h}$ following transfection. (A) Nuclear proteins were harvested, and protein expression levels of p-CREB-1 and CREB-1 were detected by western blotting. (B) Quantification of western blotting revealed that in the FSK plus TSA intervention group, expression of p-CREB-1 was significantly increased compared with the control group. (C) Collagen I mRNA expression levels were detected by reverse transcription-quantitative polymerase chain reaction. Collagen I mRNA expression levels were significantly reduced by $37 \%$ following pRSV-CREB-1 transfection in the FSK plus TSA group compared with the no treatment group. mRNA results were normalized to GAPDH expression. Data are expressed as the mean \pm standard deviation of at least three independent experiments. " $\mathrm{P}<0.05$ and ${ }^{* *} \mathrm{P}<0.01$ vs. no treatment group. CREB-1, cyclic adenosine monophosphate response element binding protein-1; FSK, forskolin; p, phosphorylated; TSA, trichostatin A.

CREB-1 significantly attenuated activation of ERK1/2 induced by TGF- $\beta 1$ ( $\mathrm{P}<0.001$; Fig. $4 \mathrm{~A}$ and $\mathrm{B})$. No statistically significant differences were observed in ERK1/2 mRNA expression levels ( $\mathrm{P}=0.453$; Fig. 4C). TGF- $\beta 1$ stimulated the expression of RhoA (Fig. 4D) and ROCK1 (Fig. 4E); however, overexpression of CREB-1 reduced RhoA and ROCK1 mRNA expression levels, weakening the effect of TGF- $\beta 1$ stimulation.

Acetylation extends the function of phosphorylated CREB-1 in decreasing expression levels of collagen $I$. The above results indicated that CREB-1 may be activated by FSK, and have a key antifibrolytic role. However, inactivated p-CREB-1 diminishes the effect of FSK stimulation. A previous study revealed that the HDAC inhibitor TSA acetylates CREB-1 and extends its phosphorylation level in an NIH/3T3 D5 cell line (13). To determine whether TSA has the same effect in HSCs, cells were stimulated with FSK and/or TSA to induce CREB-1 post-translational modification. The results demonstrated that in the FSK plus TSA group, protein expression levels of p-CREB-1 were significantly increased compared with the control group (3.6-fold greater than control group; $\mathrm{P}<0.001$; Fig. 5A and B). Differences in CREB-1 expression between the other groups was not statistically significant $(\mathrm{P}=0.139)$. In addition, following $4 \mathrm{~h}$ of treatment with FSK and TSA in the pRSV-CREB-1 group, collagen I mRNA expression levels were significantly reduced by $37 \%$ compared with the no treatment group ( $\mathrm{P}=0.011$; Fig. 5C).

\section{Discussion}

As an important member of the activating transcription factors/CREB family, CREB-1 is involved in a variety of biological functions, including inhibiting inflammation and apoptosis, enhancing immunity and memory, and regulating growth and development $(12,14)$. Previous studies have revealed that that CREB-1 has antifibrolytic effects in lung, myocardial and corneal tissue $(5,6,15)$. However, the role of CREB-1 in liver fibrosis remains to be elucidated. The results of the present study demonstrated that CREB-1 activated by FSK significantly reduces collagen I expression in HSCs. This suggested that CREB-1 exerts an antifibrolytic effect in liver tissue.

TGF- $\beta 1$ has an important role in the liver fibrotic response, and inhibition of the TGF- $\beta 1$ signaling pathway is a potential therapeutic strategy for the treatment of fibrosis. TGF- $\beta 1$ binds to TGF- $\beta 1$ receptors, which induce the phosphorylation of Smad2. Smad2 in turn phosphorylates the highly analogous protein, Smad3, referred to as R-Smad, and induces the recruitment of Smad4. The activated Smad3/4 complex translocates into the nucleus to interact with Smad-binding elements and regulate downstream genes, particularly collagen (16). By contrast, Smad7 acts in opposition to this process by binding to the type I receptor and preventing recruitment of phosphorylated Smad2 (17). TGF- $\beta 1$ activates Smad proteins, ERK1/2 and RhoA/ROCK1 $(11,18,19)$. In the present study, TGF- $\beta 1$ induced 
the phosphorylation of Smad2/3 in HSCs, as well as the phosphorylation of ERK1/2 and the expression of RhoA/ROCK1. This effect was significantly reduced following treatment with FSK. In addition, activation of CREB-1 enhanced expression of the inhibitory factor, Smad7. Notably, the combined treatment of TGF- $\beta 1$ and FSK in CREB-1-transfected HSCs resulted in a greater reduction in $\mathrm{p}$-Smad2/3 and p-ERK1/2 expression levels and a greater increase in Smad7 expression levels compared with FSK treatment alone. These results suggested that activated CREB-1 has a strong inhibitory effect on TGF- $\beta 1$-mediated activation during fibrotic responses via blocking Smad-dependent and -independent signaling pathways. However, other signaling pathways, including phosphatidylinositol-3-kinase/serine-threonine kinase, c-Jun $\mathrm{N}$-terminal kinase, mitogen-activated protein kinase and TGF- $\beta$-activated kinase 1 have been reported to be associated with fibrosis (20-23). The specific mechanism underlying CREB-1 regulation in HSCs remains to be fully elucidated.

Post-translational modifications of CREB-1 include phosphorylation, acetylation, ubiquitination, sumoylation and glycosylation (8). Phosphorylation has an important role in transcriptional activation and biological functions. Without phosphorylation, CREB-1 has almost no biological activity. However, phosphorylated CREB-1 may be hydrolyzed in internal environments. A previous study revealed that acetylation may improve the stability of phosphorylated proteins by altering the space conformation of CREB-1 and allowing its escape from hydrolysis (13). Results from the present study demonstrated that p-CREB-1 reached a peak level following 30 min of FSK treatment, then declined gradually. The previous study on cardiac fibroblasts demonstrated that the peak level was reached at $5 \mathrm{~min}$ and then declined rapidly (10). This may have been due to the experimental conditions, or the fact that the former was a cell line, whereas the latter was a primary cell (10). HSCs treated with cAMP-elevating agent or cAMP-elevating agent plus HDAC inhibitor for $4 \mathrm{~h}$ demonstrated a significant increase in the expression of Ser-133-phosphorylated CREB compared with FSK treatment alone. Furthermore, co-treatment reduced collagen I mRNA expression levels significantly in the CREB-1 overexpression group. These results suggested that a HDAC inhibitor may prolong p-CREB-1 phosphorylation at Ser-133 and enhance its antifibrolytic capacity.

CREB-binding protein (CBP) was originally identified as a co-activator of CREB. CBP acts as a bridging factor between CREB and transcription factor IIB. CBP, containing its homologue $\mathrm{p} 300$, possesses intrinsic histone acetyltransferase activity; $\mathrm{CBP} / \mathrm{p} 300$ acetylates histones and non-histone proteins, including transcription factors (24). The phosphorylation of CREB at Ser-133 leads to the association of CREB via the kinase-inducible domain with a short domain in $\mathrm{CBP}$ termed the kinase-inducible domain interacting domain; p-CREB is further acetylated, and CREB activity during the late attenuation phase is prolonged (25). However, CBP, as a broad-spectrum coactivator, directly interacts with $\mathrm{R}$-Smad-Smad4 via the C-terminal domain to activate the transcription of TGF- $\beta 1$-responsive genes (26). Therefore, CREB-1 may compete with R-Smad-Smad4 to interact with CBP. In the present study, the expression of collagen I was significantly reduced by FSK and TSA treatment following
CREB-1 overexpression, demonstrating that excessive acetylated p-CREB-1 may inhibit R-Smad-Smad4 recruitment of CBP, thus inhibiting TGF- $\beta 1$ signaling pathways. However, additional studies including in vivo experiments are required to provide further evidence for the antifibrolytic effect of acetylated p-CREB-1.

In conclusion, the results of the present study demonstrated that p-CREB-1 inhibits TGF- $\beta 1$-mediated liver fibrosis by blocking the Smad-dependent and -independent signaling pathways in HSCs. Furthermore, the results of the current study revealed that CREB-1 is not sustainably activated; however, acetylation extends CREB-1 phosphorylation and enhances its antifibrolytic effect. The results of the present study suggested that CREB-1 may be a useful potential therapeutic target for the treatment of liver fibrosis.

\section{Acknowledgements}

The present study was supported by the National Natural Science Foundation of China (grant no. 81170401).

\section{References}

1. He Y,Huang C, Sun X,Long XR,Lv XW and Li J: MicroRNA-146a modulates TGF-betal-induced hepatic stellate cell proliferation by targeting SMAD4. Cell Signal 24: 1923-1930, 2012.

2. van der Smissen A, Samsonov S, Hintze V, Scharnweber D, Moeller S, Schnabelrauch M, Pisabarro MT and Anderegg U: Artificial extracellular matrix composed of collagen I and highly sulfated hyaluronan interferes with TGF $\beta(1)$ signaling and prevents TGF $\beta(1)$-induced myofibroblast differentiation. Acta Biomater 9: 7775-7786, 2013.

3. Zhang Y, Liu P, Gao X, Qian W and Xu K: rAAV2-TGF- $\beta(3)$ decreases collagen synthesis and deposition in the liver of experimental hepatic fibrosis rat. Dig Dis Sci 55: 2821-2830, 2010.

4. Deng L, Li Y, Huang JM, Zhou GY, Qian W and Xu KS: Effects of p-CREB-1 on transforming growth factor- $\beta 3$ auto-regulation in hepatic stellate cells. J Cell Biochem 112: 1046-1054, 2011.

5. Chan EC, Dusting GJ, Guo N, Peshavariya HM, Taylor CJ, Dilley R, Narumiya S and Jiang F: Prostacyclin receptor suppresses cardiac fibrosis: Role of CREB phosphorylation. J Mol Cell Cardiol 49: 176-185, 2010.

6. Liu X, Sun SQ and Ostrom RS: Fibrotic lung fibroblasts show blunted inhibition by cAMP due to deficient cAMP response element-binding protein phosphorylation. J Pharmacol Exp Ther 315: 678-687, 2005.

7. Liu X, Sun SQ, Hassid A and Ostrom RS: cAMP Inhibits transforming growth factor-beta-stimulated collagen synthesis via inhibition of extracellular signal-regulated kinase $1 / 2$ and smad signaling in cardiac fibroblasts. Mol Pharmacol 70: 1992-2003, 2006.

8. Johannessen M, Delghandi MP and Moens U: What turns CREB on? Cell Signal 16: 1211-1227, 2004.

9. Livak KJ and Schmittgen TD: Analysis of relative gene expression data using real-time quantitative PCR and the 2(-Delta Delta C(T)) Methods. Methods 25: 402-408, 2001.

10. Husse B and Isenberg G: Cyclic mechanical strain causes cAMP-response element binding protein activation by different pathways in cardiac fibroblasts. Heart Int 5: e3, 2010.

11. Yoshida $\mathrm{K}$ and Matsuzaki K: Differential regulation of TGF- $\beta /$ Smad signaling in hepatic stellate cells between acute and chronic liver injuries. Front Physiol 3: 53, 2012.

12. Ran I, Laplante I and Lacaille JC: CREB-dependent transcriptional control and quantal changes in persistent long-term potentiation in hippocampal interneurons. J Neurosci 32: 6335-6350, 2012

13. MichaelLF, Asahara H, Shulman AI, Kraus WL and Montminy M: The phosphorylation status of a cyclic AMP-responsive activator is modulated via a chromatin-dependent mechanism. Mol Cell Biol 20: 1596-1603, 2000.

14. Wen AY, Sakamoto KM and Miller LS: The role of the transcription factor CREB in immune function. J Immunol 185: 6413-6419, 2010. 
15. Xing D and Bonanno JA: Effect of cAMP on TGFbeta1-induced corneal keratocyte-myofibroblast transformation. Invest Ophthalmol Vis Sci 50: 626-633, 2009.

16. Ding N, Yu RT, Subramaniam N, Sherman MH, Wilson C, Rao R, Leblanc M, Coulter S, He M, Scott C, et al: A vitamin $\mathrm{D}$ receptor/SMAD genomic circuit gates hepatic fibrotic response. Cell 153: 601-613, 2013.

17. Chung AC, Dong Y, Yang W, Zhong X, Li R and Lan HY: Smad7 suppresses renal fibrosis via altering expression of TGF- $\beta /$ Smad3-regulated microRNAs. Mol Ther 21: 388-398, 2013.

18. Li L, Fan D, Wang C, Wang JY, Cui XB, Wu D, Zhou Y and Wu LL: Angiotensin II increases periostin expression via Ras/p38 MAPK/CREB and ERK1/2/TGF- $\beta 1$ pathways in cardiac fibroblasts. Cardiovasc Res 91: 80-89, 2011.

19. Cho IJ, Kim YW, Han CY, Kim EH, Anderson RA, Lee YS, Lee $\mathrm{CH}$, Hwang SJ and Kim SG: E-cadherin antagonizes transforming growth factor $\beta 1$ gene induction in hepatic stellate cells by inhibiting RhoA-dependent Smad3 phosphorylation. Hepatology 52: 2053-2064, 2010.

20. Lee KS, Park SJ, Kim SR, Min KH, Lee KY, Choe YH, Hong SH, Lee YR, Kim JS, Hong SJ and Lee YC: Inhibition of VEGF blocks TGF-betal production through a PI3K/Akt signalling pathway. Eur Respir J 31: 523-531, 2008.
21. Zhang L,LiY,Chen M,Su X,YiD,Lu Pand Zhu D: 15-LO/15-HETE mediated vascular adventitia fibrosis via p38 MAPK-dependent TGF- $\beta$. J Cell Physiol 229: 245-257, 2014.

22. Ding ZY, Jin GN, Liang HF, Wang W, Chen WX, Datta PK, Zhang MZ, Zhang B and Chen XP: Transforming growth factor $\beta$ induces expression of connective tissue growth factor in hepatic progenitor cells through Smad independent signaling. Cell Signal 25: 1981-1992, 2013

23. Yang L, Inokuchi S, Roh YS, Song J,Loomba R, Park EJ and Seki E: Transforming growth factor- $\beta$ signaling in hepatocytes promotes hepatic fibrosis and carcinogenesis in mice with hepatocyte-specific deletion of TAK1. Gastroenterology 144: 1042-1054.e4, 2013.

24. Kasper LH, Lerach S, Wang J, Wu S, Jeevan T and Brindle PK: $\mathrm{CBP} / \mathrm{p} 300$ double null cells reveal effect of coactivator level and diversity on CREB transactivation. EMBO J 29: 3660-3672, 2010.

25. Wang J, Weaver IC, Gauthier-Fisher A, Wang H, He L, Yeomans J, Wondisford F, Kaplan DR and Miller FD: CBP histone acetyltransferase activity regulates embryonic neural differentiation in the normal and Rubinstein-Taybi syndrome brain. Dev Cell 18: 114-125, 2010.

26. Chen W, Lam SS, Srinath H, Schiffer CA, Royer WE Jr and Lin K: Competition between Ski and CREB-binding protein for binding to Smad proteins in transforming growth factor-beta signaling. J Biol Chem 282: 11365-11376, 2007. 